\title{
Maternité Et Risques Perçus Face Aux Examens Sanguins Et Au Test Du VIH-SIDA : Etude De Cas Dans Une Formation Sanitaire Urbaine De Bouaké (Côte D'ivoire)
}

\author{
Dr. Mazou Gnazegbo Hilaire \\ Socio-anthropologue de la santé, Enseignant-Chercheur, \\ Université Alassane Ouattara, Côte d'ivoire.
}

Doi: 10.19044/esj.2017.v13n30p139 URL:http://dx.doi.org/10.19044/esj.2017.v13n30p139

\begin{abstract}
Pregnancy is a physiological condition that requires prenatal followup during which medical care ensures that complications in childbirth are prevented and that a healthy child is born as much as possible. It is therefore important for pregnant women to undergo a number of clinical examinations, including blood tests and HIV / AIDS testing, which are in the process of being integrated into the monitoring of women at the primary health care. However, pregnant women do not systematically use these practices in Urban Sanitary Training in Côte d'Ivoire generally and in Bouaké in particular. The free choice of women to submit to the various tests, the health benefits of which are the subject of recurrent information campaigns, does not always lead to their rigorous application during prenatal consultations. It therefore appears that the resistances or the sometimes unsystematic choices of acceptance of the tests and their referents become the object of reflection in the face of the challenge of improving the safe motherhood rate and the attainment of a generation without HIV-AIDS. What are the social attitudes and perceptions related to blood tests and HIV-AIDS testing in pregnant women ? The aim of this study is to understand the social logics underlying the positive or negative attitude of pregnant women to blood tests and the HIV-AIDS test. To address this concern, an essentially qualitative study was carried out among 16 pregnant women and the medical staff of the Urban Sanitary Formation of Ahougnansou in Bouaké (Côte d'Ivoire). The main finding of this study is that attitudes of pregnant women have links to socially situated representations of the disease, stigma, education, income, and perceived risks to social and marital relationships.
\end{abstract}

Keywords : Pregnancy, Pregnancy consultation, attitude, social perception, reluctance, blood tests, HIV / AIDS, Urban health training 


\section{Résumé}

La grossesse est un état physiologique qui nécessite un suivi prénatal au cours duquel la prise en charge médicale assure de prévenir les éventuelles complications à l'accouchement ainsi que la naissance d'un enfant en parfaite santé, dans la mesure du possible. Il est de ce fait important pour la femme enceinte de se soumettre à un certain nombre d'examens cliniques, au nombre desquels les examens sanguins et le test du VIH-SIDA sont en passe d'être des pratiques intégrant le suivi des femmes au niveau de soins de santé primaire. Cependant, les femmes enceintes ne recourent pas systématiquement à ces pratiques dans les Formations Sanitaires Urbaines en Côte d'ivoire de façon générale et à Bouaké en particulier. Le libre choix laissé aux femmes de se soumettre aux différents tests dont les bienfaits sanitaires font l'objet de campagnes d'information récurrentes ne conduit pas toujours à leur application rigoureuse lors des consultations prénatales CPN. Il apparait dès lors que les résistances ou les choix parfois non systématiques de l'acceptation des tests ainsi que leurs référents deviennent les objets d'une réflexions face au défi d'améliorer le taux de maternité sans risque et de l'atteinte d'une génération sans VIHSIDA. Quelles sont les attitudes et perceptions sociales liées aux examens sanguins et au test du VIH-SIDA chez les femmes enceintes ? La présente étude, vise à comprendre les logiques sociales qui sous-tendent l'attitude favorable ou non des femmes enceintes face aux examens sanguins et au test du VIH-SIDA. Pour répondre à cette préoccupation, une étude essentiellement qualitative a été réalisée auprès de 16 femmes enceintes et du personnel médical de la Formation Sanitaire Urbaine d'Ahougnansou dans la commune de Bouaké en Côte d'Ivoire. Il ressort principalement de cette étude, que les attitudes des femmes enceintes ont des liens avec les représentations socialement situées de la maladie, la stigmatisation, le niveau d'instruction, de revenu ou encore les risques perçus des résultats sur les relations sociales et conjugales.

Mots clés : Femmes enceintes, Consultation Prénatale, attitude, perception sociale, réticence, examens sanguins, VIH-SIDA, Formation sanitaire urbaine

\section{Contexte et problématique de l'étude}

Dans les pays en développement, la mortalité maternelle, néonatale et infantile est un problème majeur de santé publique. En Côte d'Ivoire, les mortalités néonatales et infantiles sont évaluées respectivement à 41 et 84 décès pour 1000 Naissances vivantes (EIS, 2005). Le taux de mortalité maternelle est passé de 543 décès maternels pour 100000 naissances 
vivantes en 2005 (INS, EDS-CI, 1994 ; INS, EIS, 2005) à 614 décès en 2011 (INS et ICF, 2012). La mortalité maternelle est estimée à deux (2) décès toutes les trois heures, avec le VIH-SIDA comme l'une des principales causes. Le taux de transmission mère-enfant du VIH s'élève à environ $8 \%$ et la prévalence à 4,6\% (OPALD, 2014). Les femmes enceintes, bien que particulièrement vulnérables au VIH-SIDA, ne savent pas qu'un traitement approprié peut freiner la transmission mère-enfant. D'où l'importance de la consultation prénatale (CPN) qui constitue un ensemble d'activités essentielles au suivi de la grossesse, permettant d'éviter à des millions de femmes et de nouveau-nés, la mort ou des séquelles qui peuvent être permanentes (Koffi et al., 2000 ; OMS, 1994).

Cependant comme l'a souligné N'guessan (2015), il existe encore d'énormes discontinuités entre les niveaux d'utilisation des services prénataux. Ce faible recours aux structures de santé de proximité, explique en partie que dans des localités, les femmes enceintes ne réalisent pas toutes les consultations prénatales. A la Formation Sanitaire Urbaine (FSU) d'Ahougnansou (Bouaké), il ressort que les femmes enceintes observent les 4 consultations prénatales. Selon les statistiques tenues par les soins de cette FSU, plus de $96 \%$ des femmes enceintes ont suivi régulièrement les 4 Consultations Prénatales (CPN) préconisées par l'OMS, dont $80 \%$ refusent de se soumettre aux tests sanguins et de dépistage du VIH-SIDA. Un chiffre exceptionnel qui cependant questionne au moins pour deux raisons : (i) au regard du faible niveau d'usage des structures de santé (en général sur le plan national), comment s'explique l'assiduité des femmes de cette FSU dans le suivi des CPN ? et (ii) qu'est-ce qui explique la non-acceptation des test sanguins et de dépistage du VIH-SIDA chez certaines femmes usagères de cette formations sanitaires, quand se soumettre à ces tests est une tendance généralisée et observable?

Il convient alors de comprendre les justifications sociales qui soustendent l'attitude de ces femmes enceintes face à ces formalités qui permettent pourtant de garantir le bien-être de la mère et celui de l'enfant.

Au niveau socio-économique, la femme occupe une place de choix dans la société ivoirienne. D'où l'important de se pencher sur tout ce qui, de près ou de loin touche à la femme et notamment à sa santé. C'est dans cette perspective que s'inscrit le plan d'intervention de l'indication stratégique de l'OMS, contre la transmission mère-enfant, à travers le dépistage et les examens de sang. De plus, en dehors du VIH-SIDA, les examens de sang permettent de détecter d'autres maladies toutes aussi importantes qui pourraient menacer la santé de la mère et de l'enfant. C'est dans ce cadre que l'Organisation Panafricaine de Lutte pour la Santé (OPALS) a fait des orientations consacrées à la protection de la santé mère-enfant. Enfin, une étude sur les attitudes et perceptions des femmes enceintes permettrait de 
dévoiler les motivations et sentiments méconnus vis-à-vis de ces différents examens, en vue de mieux orienter les programmes de sensibilisation sur le sujet du VIH-SIDA et des examens sanguins.

\section{Matériels et méthodologie}

\section{La nature de l'étude}

Cette recherche vise à comprendre les logiques sociales qui justifient l'attitude des femmes enceintes vis-à-vis des examens sanguins et du test du VIH-SIDA à la FSU d'Ahougnansou. La méthode d'enquête qui permet de répondre à cette problématique est l'approche qualitative. Cette approche a été mobilisée parce qu'elle a le mérite de donner sens aux phénomènes à travers l'observation, la description, l'interprétation, l'appréciation du milieu et du phénomène tel qu'il se présente (N'da, $2002: 21$ ). Nous avons privilégié les entretiens individuels semi-structurés qui permettaient aux femmes enceintes de s'exprimer librement.

\section{Le site d'enquête}

L'étude s'est déroulée à la FSU d'Ahougnansou située sur la voie principale du quartier entre le cinquième arrondissement de police et le château. Cette formation sanitaire créée en 1985, a été équipée par la Compagnie Ivoirienne de Développement du Textile (CIDT). Depuis sa création, elle contribue à la prise en charge médicale des populations du quartier et celles des quartiers environnants tels que N'Dakro, Broukro, Banco, Adjéyaokro, Cité CIDT et Tchèlèkro. Malgré ses nombreuses difficultés, la formation sanitaire urbaine d'Ahougnansou, tend à assurer le suivi des femmes enceintes au niveau des examens sanguins et du test du VIH-SIDA.

\section{La sélection des enquêtés}

Dans le cadre de cette recherche, nous avons eu recours à trois types de populations cibles pour la collecte des informations. Il s'agit notamment, du médecin chef, des sages-femmes et des femmes enceintes.

La problématique de cette recherche porte sur les femmes enceintes et leurs perceptions vis-à-vis des examens sang et le test du VIH. En fonction de la réalité du terrain, nous avons utilisé la technique à choix raisonné. La disponibilité des femmes à répondre à nos questions, a été déterminante comme critère. En effet, nous nous sommes d'abord référés au médecin-chef, aux sages-femmes et à la conseillère communautaire. Avec le médecin-chef de la FSU d'Ahougnansou, il a été question d'avoir des détails sur le taux de fréquentation du centre par les femmes enceintes et sur la politique de sensibilisation incitative mis en place pour montrer le bien-fondé de ces examens. Les sages-femmes quant à elles, ont permis d'entrer en contact 
avec les femmes qui venaient en consultation, mais aussi d'obtenir le contact de celles qui n'avaient pas de rendez-vous prévu pendant la période d'enquête. La conseillère communautaire a permis de faire le point de la situation du dépistage et des examens sanguins. Enfin les femmes enceintes, sont celles qui détiennent la vérité sur leur comportement en lien avec les différentes perceptions sociales. Seules elles, peuvent expliquer les raisons profondes qui motivent leur attitude à l'égard de ces différents examens.

Nous avons ainsi, enquêté toutes les femmes enceintes qui ont bien voulu se prêter à notre guide d'entretien. Cette recherche a mobilisé au total, vingt-quatre (24) personnes dont, le médecin-chef, la conseillère communautaire, sept (6) sages-femmes et seize (16) femmes enceintes.

\section{Techniques et outils de collectes de données}

Pour cette étude, l'observation directe a permis d'apprécier les échanges entre les sages-femmes, la conseillère communautaire et les femmes enceintes. Puis, nous nous sommes appuyés sur deux types de données. Tout d'abord les données secondaires, essentiellement bibliographiques, ont permis d'avoir des connaissances sur les composantes de la consultation prénatale, de comprendre la nécessité de recours aux différents examens en vue de limiter les dangers liés à la grossesse. Le second type d'information mobilisé est constitué de données primaires collectées auprès des femmes enceintes, des sages-femmes et du médecin chef et de la conseillère communautaire. Nous entendons par données primaires, les propos et discours tenus par les personnes enquêtées et tendant à rendre compte de la façon dont elles vivent et se représentent les choses (Olivier de Sardan, 2000). Ces informations collectées à partir d'entretiens semi-directifs, visaient à informer sur les connaissances des femmes enceintes, leurs perceptions, leurs pratiques et attitudes vis-à-vis des examens de sang et du dépistage, ainsi que leurs choix stratégiques et leurs justifications.

L'étude a été menée à la FSU d'Ahougnansou entre Février et Avril 2016. Cette formation sanitaire a été choisie à cause de la disponibilité du personnel soignant et de sa situation géographique. De plus, nous avons voulu comprendre les stratégies mises en place pour attirer le maximum de femmes enceintes en provenance des quartiers environnants. Enfin, il s'agissait aussi pour nous de comprendre si les femmes étaient plus disposées à effectuer les examens sanguins et le test du dépistage dans un espace qui semble plus discret et excentré du centre-ville. L'enquête de terrain a permis d'abord d'observer directement les échanges entre les femmes enceintes, les sages-femmes et la conseillère communautaire. Ces échanges portaient sur la sensibilisation, le suivi régulier des consultations prénatales, l'importance du test du VIH et des examens sanguins et les connaissances sur le sujet d'étude. Ensuite, d'administrer le guide d'entretien 
au médecin-chef, à la conseillère communautaire, aux sages-femmes, et aux femmes enceintes. Les discours des enquêtés ont fait l'objet d'une retranscription exhaustive. Les données collectées ont par la suite été analysées par le biais de la théorie du choix raisonné empruntée à la psychologie sociale. Selon cette théorie, la décision d'un individu à adopter un comportement est déterminée par son attitude et ses normes subjectives relatives au comportement en question (Fishbein et Ajzen, 1975). Ainsi, l'attitude des femmes enceintes face aux examens sanguins et au test du VIH s'explique par les préjugés et les prénotions autour de la maladie.

\section{Resultats De La Recherche}

Consultations prénatales et stratégies de lutte contre la transmission mère-enfant du VIH-SIDA et autres maladies

Les femmes enceintes ne sont pas considérées comme des personnes malades, à moins qu'elles souffrent d'une pathologie chronique, mais pas non plus comme des personnes en bonne santé. Elles sont plutôt traitées comme des personnes à risque. Une surveillance rapprochée permet de mesurer les risques pour elles ou leur fœtus et d'agir de manière préventive ou curative (Vassy et Champenois-Rousseau, 2014). La prise de sang et la réalisation des différents examens au cours des consultations prénatales permettent de surveiller le bon déroulement de la grossesse. En effet, lors de notre enquête de terrain à la FSU d'Ahougnansou, nous avons observé que le taux de consultation prénatale (CPN) est passé de $27 \%$ en 2014 à $96 \%$ en 2015. Cette hausse s'expliquerait, selon les agents de santé (le médecin-chef et les sages-femmes) par le fait que les femmes enceintes ont un suivi régulier des rendez-vous prénataux. On constate ainsi un bon suivi des CPN par les femmes enceintes fréquentant la FSU d'Ahougnansou tel que le recommande l'OMS.

\section{Les consultations prénatales}

La consultation prénatale est la surveillance infirmière ou médicale et les soins apportés à la future mère depuis la conception jusqu'au début du travail. La consultation prénatale est une occasion pour les prestataires de permettre à la femme enceinte d'effectuer un bilan de santé, de lui fournir des soins préventifs et éventuellement un traitement et de lui apprendre des mesures à observer à domicile pour mieux suivre sa grossesse et améliorer les chances de survie de son nouveau-né (OMS, 2006).

Les consultations prénatales définissent ainsi les différents rendezvous de la femme enceinte durant toute la période de sa grossesse jusqu'à l'accouchement. Le suivi de celles-ci, est une priorité à la formation sanitaire d'Ahougnansou. Selon le médecin-chef 
"Lorsque les consultations prénatales sont bien suivies, elles permettent d'éviter certains désagréments lors de l'accouchement. Mais, bien avant, elles contribuent à la surveillance médicale de la mère et de l'enfant ».

Toujours selon le médecin-chef, ces consultations prénatales ont un double objectif :

$\checkmark \quad$ S'assurer que la grossesse évolue de façon naturelle, ce qui est et restera la règle dans la majorité des cas.

$\checkmark \quad$ Rechercher la présence ou la survenue d'éléments anormaux susceptible de transformer un état physiologique, ou un état pathologique comportant des risques pour la mère et pour l'enfant. C'est pourquoi nous préférons cette définition qui la présente comme un ensemble d'activités préventives et curatives prodiguées à une femme en fonction du terme de sa grossesse.

De façon spécifique, les consultations prénatales permettent de :

$\checkmark \quad$ Promouvoir et maintenir la santé physique, mentale et sociale de la mère et du fœtus par le biais de l'éducation sur la nutrition, l'hygiène personnelle et l'accouchement.

$\checkmark \quad$ Dépister et prendre en charge les complications pendant la grossesse, qu'elles soient médicales, chirurgicales ou obstétricales.

$\checkmark \quad$ Former un plan de préparation à la naissance et des mesures à prendre en cas de complications.

$\checkmark \quad$ Aider à préparer la mère à allaiter de manière, à vivre un post-partum normal et à prendre soin de l'enfant physiquement, psychologiquement et socialement.

$\checkmark \quad$ Formuler des conseils centrés sur la mère aux femmes, partenaires et familles en fonction de l'âge gestationnel.

Le personnel soignant de la formation sanitaire urbaine d'Ahougnansou s'inscrit résolument dans cette perspective. Les médecins et les sages-femmes s'attèlent à mettre tout en œuvre pour satisfaire les femmes enceintes et les orienter davantage lors des CPN.

\section{Les stratégies de luttes contre la transmission mère-enfant du VIH- SIDA et autres maladies}

Les consultations prénatales et les différents examens sont directement inscrits dans les thèmes d'information, éducation et communication (IEC) et de communication pour le changement de comportement (CCC). Les femmes enceintes ont reçu des conseils sur les bienfaits du bon suivi des CPN à travers des sensibilisations répétées lors de leurs différents rendez-vous à la formation sanitaire urbaine d'Ahougnansou. Ces stratégies de sensibilisation ont eu un impact positif sur le taux de participation aux différentes CPN qui est passé de $27 \%$ en 2014 à $96 \%$ en 
2015. Au cours de ces rencontres, les prestataires de soins mettent un accent particulier sur l'importance du test de dépistage du VIH-SIDA et sur tous les autres examens sanguins. Le but étant d'inciter les femmes enceintes à se faire consulter davantage. La sensibilisation porte sur les éventuels dangers liés à une grossesse non suivie, les risques auxquels s'exposent le fœutus et la femme enceinte dont on ignore la sérologie et les autres maladies que les examens sanguins peuvent permettre d'identifier. Selon certaines femmes enceintes, ces informations leurs étaient régulièrement données en vue de leur montrer l'importance de ces tests. Comme le dit F., une des femmes enceintes enquêtée :

«Tous les matins, quand nous nous rendons à la FSU d'Ahougnansou, nous sommes sensibilisées par la conseillère communautaire sur le VIH-SIDA à savoir les dangers qui guettent notre santé et celle de notre futur bébé si nous ne connaissons pas notre statut sérologique ».

Malgré la sensibilisation répétée, certaines femmes enceintes ne se soumettent pas systématiquement au dépistage et aux différents examens comme l'atteste la conseillère communautaire.

"Je me rendais chez certaines femmes qui étaient réticentes afin de les amener à se faire dépister ».

Les sages-femmes à leur tour, sensibilisaient les femmes enceintes au respect des différents rendez-vous et le suivi de tous les examens en vue du bon déroulement de la grossesse. Comme le confirme cette enquêtée.

"Plus facile pour une femme de se soumettre à un examen et de mieux coopérer quand elle est renseignée non seulement sur l'importance mais aussi sur les dangers. Encore plus quand elle peut avoir une signification des actes qu'elle doit subir. " (K, Sagefemme).

Plusieurs femmes enceintes ont reconnu avoir reçu de nombreux conseils et sensibilisations sur le test de dépistage au VIH et l'importance des examens sanguins. Ce qui les a motivés à se faire consulter d'avantages et à être fidèles aux différents rendez-vous. C'est le cas de cette dame qui dit ceci ;

"Tout le temps quand nous nous rendons à la FSU d'Ahougnansou soit la conseillère communautaire ou quelques sages-femmes nous parlent du dépistage au VIH et des examens sanguins. C'est ce qui a fait que j'ai décidé de faire le test du SIDA. Parce que je veux que mon enfant se porte bien et moi aussi. Je ne veux pas de vilaines maladies. C'est bon de savoir ce qu'on a et puis eux (prestataires de santé), ils connaissent leur travail. ") (V, femme enceinte). 
La sensibilisation répétée et adaptée au test de dépistage et aux autres examens sanguins encourage les femmes enceintes à connaitre leur statut sérologique.

Attitudes et perceptions sociales des femmes enceintes vis-à-vis des examens sanguins et du test de VIH-SIDA.

L'attitude des femmes enceintes face aux différents examens de sang varie d'une femme à une autre. Plusieurs raisons profondes justifient les différentes attitudes adoptées par ces femmes enceintes. Il s'agit entre autres des ressources financières et la peur d'être stigmatisée.

\section{La difficulté financière comme une entrave aux examens sanguins et au test du VIH-SIDA}

Les problèmes financiers constituent une entrave à la réalisation des différents examens préconisé dans le cadre des consultations prénatales, pour les enquêtées qui sont pour la plupart, sans revenu. En effet, si le test du VIH se réalise gratuitement, il n'en est pas de même pour les autres examens que les femmes estiment trop couteux. Selon les enquêtées, le cout de ces examens varie entre 10.000 FCFA et 15.000 FCFA. Malgré le bien-fondé de ces différents examens, les femmes enceintes ne les réalisent pas systématiquement faute de moyens financiers. C'est le cas de ces femmes enceintes.

«Ces examens sont trop chers pour moi, je n'ai pas d'argent $»(\mathrm{~A}$, femme en enceinte)

«Je sais que c'est important pour moi et pour mon bébé mais Il n'y a pas d'argent et puis ça coute trop chers » (V, femme en enceinte)

\section{La dépendance de la femme enceinte vis-à-vis du conjoint}

Dans la majorité des cas, les conjoints s'opposent aux différents examens de sang préconisés dans le cadre des consultations prénatales. Cette situation empêche énormément les femmes enceintes de se soumettre à ces examens surtout celui du VIH. Ainsi, par respect pour leurs partenaires, certaines femmes refusent de faire les examens sanguins au risque de perdre leur foyer. Comme le témoigne $\mathrm{M}$.

"Quand j'ai dit à mon mari que je dois faire le test de SIDA, il s'est fâché. Il m'a dit que je ne dois pas le faire, parce que nous ne sommes pas malades. On vit dans un quartier qui est comme un village. On se connait tous, si jamais, on apprend qu'on a une maladie bizarre comme le SIDA, les gens vont nous regarder mal. $C$ 'est lui le chef de famille, donc je respecte ce qu'il dit ».

Dans la même logique. G., une jeune étudiante affirme : 
" Je connais l'importance des examens sanguins et du test $d u$ VIH-SIDA. C'est vrai que mon homme n'est pas d'accord avec moi, mais, j'ai fait le test de dépistage du VIH-SIDA sans lui dire, et il ne sait toujours pas. Mais, pour les autres, il n'y a pas d'argent. (...) si j'avais l'argent, je les ferais tous 》

Il ressort donc que les conjoints influencent considérablement les décisions des femmes enceintes vis-à-vis des différents examens de sang.

\section{La perception des risques comme frein à la réalisation des examens sanguins}

L'enquête de terrain a montré que certaines femmes enceintes, reconnaissent n'avoir aucune connaissance des risques auxquels elles sont exposées en refusant le test de dépistage du VIH et les autres examens de sang. C'est le cas respectivement de $\mathrm{T}$ et de $\mathrm{K}$, deux femmes enceintes analphabètes.

"SIDA là même, c'est quoi? On parle à la télé, à l’hôpital ici, je vois les gens parler de ça, mais, je ne peux pas expliquer à quelqu'un. Moi-même, je ne comprends pas »(T, femme enceinte).

"C'est quand je suis venue à l'hôpital ici que j'ai entendu parler de ça bien. Comment on attrape tout ça là, mais je ne connais pas trop. Et puis, les sages-femmes là disent de faire examen de sang, je ne suis pas malade, je suis enceinte. C'est pour faire quoi? Mon enfant va bien " (K, femme enceinte)

De plus, les femmes enceinte ont tendance à assimiler tout examen de sang au test de VIH-SIDA vu comme une maladie incurable. Selon M. une des femmes enquêtées :

" Je ne veux pas être victime de cette injustice et mourir de chagrin pour une maladie que je n'ai même pas » (M, femme enceinte).

En refusent les examens sanguins et le test du VIH-SIDA, les femmes enceintes s'enferment dans une réalité construite. Le repli sur soi devient alors pour ces femmes, une forme de défense et d'autoprotection.

\section{Analyse et discussion}

La consultation prénatale tient un rôle important dans le développement de la grossesse selon le personnel soignant de la formation sanitaire urbaine d'Ahougnansou. En effet, les visites prénatales sont une occasion pour les prestataires soins, de permettre à la femme enceinte d'effectuer un bilan de santé, de lui fournir des soins préventifs et éventuellement un traitement, ainsi que les mesures à observer à domicile pour mieux suivre sa grossesse et améliorer les chances de survie de son 
nouveau-né (Tiembré et al, 2010). Malgré le bien-fondé de ces consultations prénatales, les différentes campagnes de sensibilisation semblent ne pas avoir de grandes influences sur les femmes enceintes qui refusent encore de réaliser certains examens sanguins notamment le test du VIH-SIDA (N'guessan, 2015). Cette situation pourrait être sous-tendue par plusieurs facteurs. Selon Ilboudo (1996) la discontinuité s'explique par le fait que certaines femmes ignorent le nombre légal de consultation prénatale pendant la grossesse. L'ethnie, le degré de modernité, la perception de la distance et le niveau de vie du ménage sont des déterminants importants dans les comportements des femmes vis-à-vis des consultations prénatales (Kochou et Rwenge, 2014). Pour Baki (2002), le manque d'information était l'une des causes de méconnaissance des facteurs de risque, du calendrier et des avantages de la consultation prénatale. Quant à Traoré.et Coll. (2007), le niveau d'étude serait une source d'incompréhension des informations donc de méconnaissance du suivi prénatal.

Si le suivi des consultations prénatales se présente comme une préoccupation de santé publique, il n'en demeure pas moins des stratégies de lutte contre la transmission mère-enfant et les autres complications liées à la grossesse.

L'observation directe a montré que le personnel soignant de la formation sanitaire urbaine d'Ahougnansou déroulent plusieurs stratégies pour lutter contre la transmission mère-enfant dans le cadre des consultations prénatales. Il s'agit notamment de, l'information, l'éducation, la communication et les conseils qui permettent de mettre en avant les dangers auxquels les femmes enceintes sont exposées. Ainsi, le discours des prestataires de soins orienté vers les mères influence leur conduite vis-à-vis des examens et du test de VIH. Dans le cas des femmes enceintes, le discours des prestataires de santé de par l'assurance qu'il inspire, construit le point de vue des femmes. Les maladies comme le VIH-SIDA étant entourées de beaucoup de préjugés qui influencent le comportement des gestantes (Oga et al., 2014). Les praticiens amènent donc quelques femmes enceintes à accepter le dépistage par le biais de la communication qui participe à inspirer une certaine confiance (Champenois-Rousseau, 2014). Contrairement, à Damiba (2007), qui affirme que les agents de santé n'encouragent pas les femmes à se faire dépister lors des consultations prénatales. Selon cet auteur, les prestataires de soins ne jouent pas le rôle de conseillers et d'assistants. Les campagnes d'information et d'éducation sur le VIH n'ont aucun impact sur la majorité des femmes enceintes qui ignorent encore la transmission mère-enfant (Cartou et al., 1999). Ainsi, le rôle d'assistant joué par les prestataires de santé auprès des femmes enceintes ne semble pas toujours perceptible. C'est justement dans cette perspective que s'inscrit notre recherche portant sur les femmes enceintes d'Ahougnansou. Ces femmes en 
question, semblent attentives et disposées à écouter les différents messages de sensibilisation. Mais, les résultats obtenus sont en deçà de ceux escomptés, vu que les visites à domicile de la conseillère communautaire et les techniques utilisées par les sages-femmes se confrontent au refus de certaines femmes enceintes à réaliser les examens sanguins et le test du VIH. Lors de nos entretiens, quelques femmes enceintes ont admis avoir fait les examens sanguins et le test de VIH. Ce qui montre que toutes les femmes concernées, n'ont pas la même perception des consultations prénatales et des examens sanguins. Les perceptions autour de ces examens sont diverses et répondent aux réalités de chacune d'entre elles. Il ressort donc que Plusieurs raisons profondes justifient les différentes attitudes adoptées par ces femmes enceintes. Il s'agit entre autres, des difficultés financières, de l'environnement social incarné par le conjoint et la perception des risques liés aux examens sanguins et au VIH-SIDA. Pour ces femmes enceintes, le refus des examens est fortement lié au manque de ressources financières. Les conjoints trouvent souvent que les examens médicaux sont trop couteux et inutiles. Cette dépendance économique et sociale des femmes vis-à-vis des partenaires les rend vulnérables (Rebouha, 2007). De plus, la cherté des soins constitue un frein important pour les jeunes couples dont la dépendance économique et structurelle aux chefs de familles ou de clans principalement en milieu rural reste très forte (N'diaye, 2005). Même si le choix de faire ces examens demeure libre pour ces femmes, très souvent, il est sous-tendu par la dépendance financière vis-à-vis du conjoint ou même d'une tierce personne.

Le point de vue du partenaire apparait aussi comme un point déterminant dans la volonté de faire ces examens, dans la mesure où la décision de l'époux permet d'éviter des soucis conjugaux et d'entretenir la stabilité du couple, Le refus dans ce cas devient une opportunité pour ne pas être confrontée à une réalité peu souhaitable (Oga et al, 2014). Les principales raisons de refus du test du VIH, sont entre autres l'angoisse liée à la séropositivité et à la mort vécue comme certaine, la peur du rejet et la stigmatisation par l'encourage (Coulibaly et al.,1998). Le risque de la maladie n'est pas perçu. Cependant la peur et la souffrance morale liée à la connaissance d'un éventuel statut sérologique ou d'autres maladies pouvant anticiper leur mort, apparait comme un élément auquel, toutes sont attachées. Cette peur crée une psychose chez ces femmes enceintes et contraint certaines à s'abstenir. La perception du risque au plan sanitaire et social par la femme enceinte conditionne son adhésion ou non à la réalisation des examens sanguins et surtout celui du VIH-SIDA. 


\section{Conclusion}

Cette recherche a pour objectif de comprendre les logiques sociales qui justifient l'attitude des femmes enceintes vis-à-vis des examens sanguins et du test du VIH-SIDA à la Formation Sanitaire Urbaine d'Ahougnansou. Pour atteindre cet objectif, la démarche méthodologique a privilégié l'approche qualitative pour la collecte des données. Il ressort principalement de cette recherche, que le personnel soignant de la formation sanitaire urbaine d'Ahougnansou s'engage de façon régulière dans la sensibilisation par la communication pour encourager les femmes enceintes à suivre régulièrement les consultations prénatales. Mais, ces stratégies incitatives, mises en place par la structure, ne rencontrent pas toujours un succès auprès de ces femmes. La communication pour le changement de comportement se heurte au refus des femmes enceintes dans bien des cas. Cette attitude des femmes enceintes est motivée par les difficultés financières, l'environnement social incarné par le conjoint et la perception des risques liés aux examens sanguins et au VIH-SIDA.

\section{References:}

1. Baki M., 2002, «Déterminants du faible taux de dépistage de la grossesse à risque dans le district sanitaire de Boulsa », Mémoire ENSP, 55 pages.

2. Cartou M. et AL., 1999, «Attitudes des femmes enceintes vis-à-vis du dépistage VIH : l'expérience d'Abidjan et de Bobo-Dioulasso », Revue critique de l'actualité scientifique internationale sur le VIH et les virus des hépatites, $\mathrm{n}^{\circ} 1$, consulté le 13 juillet 2016.

3. Coulibaly D. et Al, 1998, «Attitudes et comportements des femmes enceintes face au dépistage du VIH à Abidjan (Côte d'Ivoire) en 1996 : Raisons du refus du test et indifférence face aux résultats. » Fonds documentaires ORSTROM, Cahiers santé $\mathrm{n}^{\circ} 8$ : 234-8.

4. Damiba J. D., 2007, «Etude des facteurs limitant l'adhésion des femmes enceintes au conseil dépistage volontaire dans le district sanitaire de Kompela. »)

5. Fishbein M.A. et Ajzen I., 1975, « Belief, attitude, intention and behavior: an introduction to theory and research » Reading, MA, Addison Wesley.

6. Ilboudo.Z.A., 1996, « Analyse de la pratique des soins prénatals dans les centres de SMI en milieu rural », Ouagadougou.

7. Ins et Icf, International, 2012, «Enquête Démographique de Santé et à Indicateurs Multiples de la Côte d'Ivoire 2011 », Calverton, Maryland.

8. Ins, 1994, «Enquête démographique et santé en Côte d'Ivoire », Abidjan. 
9. INS, 2005. «Enquête sur les Indicateurs du SIDA, Côte d'Ivoire » Calverton, Maryland, USA.

10. Kochou S.H.A. et Rwenge M.J.R., 2014, «Facteurs sociaux de la non-utilisation des services de soins prénatals ou de leur utilisation inadéquate en Côte d'Ivoire », Africain Evaluation Journal 2(1), 12 pages.

11. N'da P., 2002, « Méthodologie de la recherche : de la problématique à la discussion des résultats », Abidjan : EDUCI.

12. N'diaye P., 2005, «Déterminants socioculturels du retard de la première consultation prénatale dans un district sanitaire au Sénégal », Santé publique vol.17, n4 pp 531-538

13. N'guessan K. H., 2015, «Croyances collectives et discontinuité du suivi prénatal en milieu urbain ivoirien (Abidjan / Côte d'Ivoire) », European Scientific Journal, vol.11, No.21.

14. Oga M. et Al., 2014, «Acceptabilité du test VIH proposé aux nourrissons dans les services pédiatriques, en Côte d'Ivoire, Significations pour la couverture du diagnostic pédiatrique ", SAHARA J:Taylor \& Francis 11(1): 148-157.

15. Olivier De Sardan, J.-P. 2000, « Rendre compte des points de vue des acteurs : principes méthodologiques de l'enquête de terrain en sciences sociales », Les enquêtes participatives en débat : ambition, pratiques et enjeux, P. Lavigne Delville, N.-E. Sallamna, \& M. Mathieu, éd., Karthala ; Gret ; Icra, Paris ; Montpellier.

16. OMS, 1994, « Le dossier mère enfant : guide pour une maternité sans risque $»$, Genève.

17. OMS, 2005, « Rapport sur la santé dans le monde OMS consultations prénatales », Genève.

18. Tiembre et A1., 2010, «Évaluation de la qualité des consultations prénatales dans le district sanitaire de Grand Bassam (Côte d'Ivoire) ", Santé Publique, 2 (22), pp-221-228.

19. Traore.Y. et col., 2007, «Aspects sociodémographiques et pronostiques des grossesses non suivies chez admises dans les services de gynécologie obstétrique de l'hôpital Gabriel TOURE ». Mali.

20. Vassy C. et Champenois-Rousseau B., 2014, «Comment le risque devient une évidence : praticiens, femmes enceintes et dépistage prénatal de la trisomie $21 »$, Socio-anthropologie [En ligne], consulté le 04 janvier 2016. 